PROCEEDINGS OF THE

AMERICAN MATHEMATICAL SOCIETY

Volume 125, Number 6, June 1997, Pages 1771-1777

S 0002-9939(97)03761-1

\title{
ITERATION OF COMPACT HOLOMORPHIC MAPS ON A HILBERT BALL
}

\author{
CHO-HO CHU AND PAULINE MELLON
}

(Communicated by Palle E. T. Jorgensen)

\begin{abstract}
Given a compact holomorphic fixed-point-free self-map, $f$, of the open unit ball of a Hilbert space, we show that the sequence of iterates, $\left(f^{n}\right)$, converges locally uniformly to a constant map $\xi$ with $\|\xi\|=1$. This extends results of Denjoy (1926), Wolff (1926), Hervé (1963) and MacCluer (1983). The result is false without the compactness assumption, nor is it true for all open balls of $J^{*}$-algebras.
\end{abstract}

\section{INTRODUCTION}

There has been extensive literature on the subject of iterating holomorphic functions since the early works of Julia [14], Fatou [6], [7], Denjoy [3] and Wolff [23], [24]. We refer to [2], [20] for some interesting surveys and references.

Given a fixed-point-free holomorphic map $f: \Delta \rightarrow \Delta$ where $\Delta=\{z \in \mathbb{C}:|z|<$ $1\}$, Wolff's theorem [24] states that there is a boundary point $u \in \partial \Delta$ such that every closed disc internally tangent to $\Delta$ at $u$ is invariant under the iterates of $f$. From this follows the result of Denjoy [3] and Wolff [23] that the iterates, $f^{n}=\underbrace{f \circ \cdots \circ f}_{n}$, of $f$ converge to $u$ uniformly on compact subsets of $\Delta$. Wolff's theorem has been extended to Hilbert balls [8], and the convergence result of Denjoy and Wolff also extends to the open unit ball of $\mathbb{C}^{n}[13]$, [15], as well as some other domains in $\mathbb{C}^{n}$ [1]. Nevertheless, the convergence result fails for infinite dimensional Hilbert balls and Stachura [18] has given an example to show that it fails even for biholomorphic self-maps.

Recently, Wolff-type theorems have been established for compact holomorphic self-maps of the open unit balls of $J^{*}$-algebras (which include $C^{*}$-algebras and Hilbert spaces) [6], [25]. A natural question is whether a Denjoy-Wolff-type convergence result for compact holomorphic maps on $J^{*}$-algebras might also follow from these Wolff-type theorems. We show that this is the case for Hilbert spaces, but not the case even for finite-dimensional $C^{*}$-algebras. We prove the following result.

Theorem. Let $H$ be a Hilbert space with open unit ball $B$. Let $f: B \rightarrow B$ be a compact holomorphic map with no fixed point in $B$. Then there exists $\xi \in \partial B$ such that the sequence $\left(f^{n}\right)$ of iterates of $f$ converges locally uniformly on $B$ to the constant map taking value $\xi$.

Received by the editors December 27, 1995.

1991 Mathematics Subject Classification. Primary 46G20, 32A10, 32A17; Secondary 32M15.

(C) 1997 American Mathematical Society 
We will give a simple example to show that the above result is false if $H$ is replaced by a $C^{*}$-algebra. We also note that it has been shown in [10] that if $f: B \rightarrow B$ is fixed-point-free and so-called firmly holomorphic, then the iterates $\left(f^{n}\right)$ converge pointwise to a boundary point $\xi \in \partial B$.

It may be useful to recall the Earle-Hamilton Theorem [5] which states that every holomorphic map $f: B \rightarrow B$, where $B$ is a bounded domain in a Banach space, has a fixed-point if $f(B)$ is strictly contained in $B$.

\section{Preliminaries}

All Banach spaces will be complex. Given bounded domains $D$ and $D^{\prime}$ in any Banach spaces, we denote by $H\left(D, D^{\prime}\right)$ the space of all holomorphic maps $f: D \rightarrow$ $D^{\prime}$. We write $H(D)$ for $H(D, D)$. Every nonempty open ball $B$ in $D$ induces a norm $\|\cdot\|_{B}$ on $H\left(D, D^{\prime}\right)$ where $\|f\|_{B}=\sup _{x \in B}\|f(x)\|$ for $f \in H\left(D, D^{\prime}\right)$. The topology of local uniform convergence on $H\left(D, D^{\prime}\right)$ is the topology induced by the norms $\|\cdot\|_{B}$ where $B$ is an open ball in $D$ satisfying $\operatorname{dist}(B, \partial D)>0, \partial D$ being the boundary of $D$. Using Hadamard's three circles theorem, it has been shown in [21], [22] (see also [19, Lemma 13.1]) that $\|\cdot\|_{B_{1}}$ and $\|\cdot\|_{B_{2}}$ induce the same topology for any open balls $B_{1}, B_{2}$ in $D$ satisfying $\operatorname{dist}\left(B_{1}, \partial D\right)>0$ and $\operatorname{dist}\left(B_{2}, \partial D\right)>0$. It follows that a sequence $\left(f_{n}\right)$ in $H(D, \bar{D})$ converges to $f \in H(D, \bar{D})$ locally uniformly if, and only if, for every $x \in D,\left(f_{n}\right)$ converges uniformly to $f$ on some open ball $B$ containing $x$ and satisfying $\operatorname{dist}(B, \partial D)>0$. Given any $x$ in a Banach space $X$, and $r>0$, we let $B(x, r)=\{y \in X:\|y-x\|<r\}$. A map $f: D \rightarrow D^{\prime} \subset X$ is called compact if the closure $\overline{f(D)}$ is compact in $X$.

Lemma 1. Let $B$ be the open unit ball of a Banach space $X$ and let $f: B \rightarrow B$ be a compact holomorphic map. Then the sequence $\left(f^{n}\right)$ of iterates of $f$ has a subsequence converging locally uniformly to a function in $H(B, \bar{B})$.

Proof. Choose a sequence $\left(r_{n}\right)$ in $(0,1)$ such that $r_{n} \uparrow 1$ and $f(B) \cap B\left(0, r_{1}\right) \neq \emptyset$. We have $f(B)=\cup_{n=1}^{\infty}\left(f(B) \cap B\left(0, r_{n}\right)\right)$. We first find a subsequence of $\left(f^{n}\right)$ converging uniformly on $f(B) \cap B\left(0, r_{1}\right)$. By compactness of $\overline{f(B) \cap B\left(0, r_{1}\right)} \subset \overline{f(B)}$, there is a countable set $\left\{z_{n}\right\}$ in $f(B) \cap B\left(0, r_{1}\right)$, which is dense in $\overline{f(B) \cap B\left(0, r_{1}\right)}$.

Since $f$ is compact, $\left(f^{n}\right)$ has a subsequence, $\left(f^{(n, 1)}\right)$, such that $\left(f^{(n, 1)}\left(z_{1}\right)\right)$ converges. Likewise, $\left(f^{(n, 1)}\right)$ has a subsequence $\left(f^{(n, 2)}\right)$ such that $\left(f^{(n, 2)}\left(z_{2}\right)\right)$ converges. Proceed to find subsequences $\left(f^{(n, k)}\right)_{n}$ which converge at $z_{1}, \ldots, z_{k}$. We show that the diagonal sequence $\left(f^{(k, k)}\right)$ converges uniformly on $f(B) \cap B\left(0, r_{1}\right)$. It suffices to show that it is uniformly Cauchy on $f(B) \cap B\left(0, r_{1}\right)$. Let $\varepsilon>0$. Since $\operatorname{dist}\left(B\left(0, r_{1}\right), \partial B\right)=1-r_{1}>0$, we have

$$
\|h(z)-h(w)\| \leq \frac{\|z-w\|}{1-r_{1}}
$$

for $h \in H(B)$ and $z, w \in B\left(0, r_{1}\right)$ (cf. [19, 1.17]). By compactness, there exist $z_{n_{1}}, \ldots, z_{n_{l}}$ in $\left\{z_{n}\right\}$ such that

$$
\overline{f(B) \cap B\left(0, r_{1}\right)} \subset \bigcup_{i=1}^{l} B\left(z_{n_{i}}, \frac{\varepsilon}{3}\left(1-r_{1}\right)\right) .
$$

There exists $N$ such that $j, k>N$ implies

$$
\left\|f^{(j, j)}\left(z_{n_{i}}\right)-f^{(k, k)}\left(z_{n_{i}}\right)\right\|<\frac{\varepsilon}{3}
$$


for $i=1, \ldots, l$. Hence, for any $z \in f(B) \cap B\left(0, r_{1}\right)$, we have $z \in B\left(z_{n_{i}}, \frac{\varepsilon}{3}\left(1-r_{1}\right)\right)$ for some $i$, and

$$
\begin{aligned}
\left\|f^{(j, j)}(z)-f^{(k, k)}(z)\right\| & \leq\left\|f^{(j, j)}(z)-f^{(j, j)}\left(z_{n_{i}}\right)\right\|+\left\|f^{(j, j)}\left(z_{n_{i}}\right)-f^{(k, k)}\left(z_{n_{i}}\right)\right\| \\
& +\left\|f^{(k, k)}\left(z_{n_{i}}\right)-f^{(k, k)}(z)\right\| \\
& <\frac{\varepsilon\left(1-r_{1}\right)}{3\left(1-r_{1}\right)}+\frac{\varepsilon}{3}+\frac{\varepsilon\left(1-r_{1}\right)}{3\left(1-r_{1}\right)}=\varepsilon
\end{aligned}
$$

whenever $j, k>N$. This shows that $\left(f^{(k, k)}\right)$ is uniformly convergent on $f(B) \cap$ $B\left(0, r_{1}\right)$.

We repeat the diagonal process as follows. Choose a subsequence $\left(f^{n_{1}}\right)$ of $\left(f^{n}\right)$ converging uniformly on $f(B) \cap B\left(0, r_{1}\right)$. Then choose a subsequence $\left(f^{n_{2}}\right)$ of $\left(f^{n_{1}}\right)$ converging uniformly on $f(B) \cap B\left(0, r_{2}\right)$, and so on. The diagonal sequence $\left(f^{n_{n}}\right)$ then converges uniformly on $f(B) \cap B\left(0, r_{k}\right)$ for $k=1,2, \ldots$.

Finally, we show that $\left(f^{n_{n}+1}\right)$ converges locally uniformly on $B$. Pick $x \in B$ and choose $r, R>0$ such that $r+R=1-\|x\|$ and $\frac{r}{R}<1-\|f(x)\|$. Then $B(x, r)$ and $B\left(f(x), \frac{r}{R}\right)$ are contained in $B$. As in $(1)$, $\operatorname{dist}(B(x, r), \partial B) \geq R>0$ implies

$$
f(B(x, r)) \subset B\left(f(x), \frac{r}{R}\right) \cap f(B) \subset B\left(0, r_{k}\right) \cap f(B)
$$

for some $k$. It follows that $\left(f^{n_{n}}\right)$ converges uniformly on $f(B(x, r))$ and hence $\left(f^{n_{n}+1}\right)$ converges uniformly on $B(x, r)$.

Remark 1 . The above proof implies that every subsequence $\left(f^{n_{k}}\right)$ of the iterates $\left(f^{n}\right)$ has a locally uniformly convergent subsequence.

We need the following version of the maximum modulus principle and we include a proof for completeness (cf. [4, p.95]).

Lemma 2. Let $D$ be a domain in a Banach space $X$ and let $B$ be the open unit ball of a Hilbert space $H$. Given any holomorphic function $f: D \rightarrow \bar{B}$, we have either $f(D) \subset B$ or $f(z)=\xi \in \partial B$ for all $z \in D$.

Proof. Suppose $f\left(z_{0}\right)=\xi \in \partial B$ for some $z_{0} \in D$ where $D$ contains some open ball $B\left(z_{0}, r\right)$ with $r>0$. We show that $f(z)=\xi$ for all $z \in D$. It suffices to show $f(v)=\xi$ for all $v \in B\left(z_{0}, r\right)$. Fix $v$ arbitrary in $B\left(z_{0}, r\right)$. Define $\varphi_{v}: \Delta \longrightarrow \mathbb{C}$ by

$$
\varphi_{v}(\lambda)=\left\langle f\left(z_{0}+\lambda\left(v-z_{0}\right)\right), \xi\right\rangle \quad(\lambda \in \Delta)
$$

where $\langle\cdot, \cdot\rangle$ denotes the inner product on $H$. Then $\varphi_{v}: \Delta \rightarrow \bar{\Delta}$ and $\varphi_{v}(0)=1$ imply $\varphi_{v} \equiv 1$ by the maximum modulus principle. It follows that $f\left(z_{0}+\lambda\left(v-z_{0}\right)\right)=\xi$ for all $\lambda \in \Delta$ which gives $f(v)=\xi$ by continuity.

\section{Denjoy-Wolff-Type Result}

In this section, we prove the Theorem and give some simple examples. Wolff's theorem has been extended to fixed-point-free holomorphic self-maps $f$ of a Hilbert ball $B$, in which case there exists $\xi \in \partial B$ such that the "ellipsoids"

$$
E(\xi, \lambda)=\left\{x \in B: \frac{|1-\langle x, \xi\rangle|^{2}}{1-\|x\|^{2}}<\lambda\right\} \quad(\lambda>0)
$$

are invariant under $f$, and further, $\overline{E(\xi, \lambda)} \cap \partial B=\{\xi\}$ (cf. [8]). If $\operatorname{dim} B<\infty$, then the iterates $f^{n}$ must converge locally uniformly to the "Wolff point" $\xi$ (cf. $[13,15]$ ). 
As remarked before, the latter result is false in infinite dimensions by Stachura's example [18].

Let $\mathcal{L}(H, K)$ be the Banach space of bounded linear operators between Hilbert spaces $H$ and $K$. A closed linear subspace $Z \subset \mathcal{L}(H, K)$ is called a $J^{*}$-algebra if $T T^{*} T \in Z$ whenever $T \in Z$, where $T^{*}$ denotes the adjoint of $T$ (cf. [11, 19]). Every Hilbert space $H=\mathcal{L}(\mathbb{C}, H)$ is a $J^{*}$-algebra, and so is every $C^{*}$-algebra.

Let $B$ be the open unit ball of a $J^{*}$-algebra $Z$ and let $f: B \rightarrow B$ be a fixedpoint-free compact holomorphic map. A Wolff-type result has been obtained in [16, Theorem 5] which states that under certain conditions on $f$, there exist a "Wolff point" $\xi \in \partial B$ and circular domains $D_{z, \xi}(z \in B)$ invariant under $f$ (see also [25]). The question of whether the iterates $f^{n}$ would converge to $\xi$ was unanswered in [16]. The following example gives a negative answer.

Example 1. Let $Z=\mathbb{C} \times \mathbb{C}$ be equipped with the coordinatewise product and norm $\|(z, w)\|=\max (|z|,|w|)$. Then $Z$ is a $C^{*}$-algebra with open unit ball $\Delta \times \Delta$. Pick any fixed-point-free $h \in H(\Delta)$. Define $f: \Delta \times \Delta \rightarrow \Delta \times \Delta$ by

$$
f(z, w)=(i z, h(w)) \quad(z, w \in \Delta) .
$$

Then $f$ is fixed-point-free and we have

$$
f^{n}(z, w)=\left(i^{n} z, h^{n}(w)\right)
$$

where $\left(h^{n}\right)$ converges locally uniformly on $\Delta$ to some $\xi \in \partial \Delta$. The iterates $\left(f^{n}\right)$ clearly do not converge to any boundary point in $\partial(\Delta \times \Delta)$.

Nevertheless, we can still derive a Denjoy-Wolff-type convergence result for compact holomorphic maps on Hilbert spaces, by adapting MacCluer's arguments for $\mathbb{C}^{n}$ in [15]. A crucial step in the proof depends on the fact that the automorphisms of a Hilbert ball map affine sets to affine sets, and consequently that the fixed-point set of a nonconstant holomorphic map is affine. In contrast, the automorphisms of the open unit ball of an arbitrary $J^{*}$-algebra may distort the affine sets and the fixed-point set of a nonconstant holomorphic map need not be affine, even in the simple case of the bidisc as shown by the example below. This is one reason why a Denjoy-Wolff-type result fails for arbitrary $J^{*}$-algebras.

Let $B$ be the open unit ball of a Banach space $X$. By an affine subset of $B$ we mean a nonempty set of the form $(c+L) \cap B$ where $c \in X$ and $L$ is a closed linear subspace of $X$. If $X$ is a Hilbert space, $c$ can be chosen to be orthogonal to $L$, and also, for nonconstant $h \in H(B)$, its fixed-point set Fix $(h)=\{x \in B: h(x)=x\}$ is affine by [12] (see also [9, Theorem 23.2]). This was proved in [17] in finite dimensions.

Example 2. Let $Z=\mathbb{C} \times \mathbb{C}$ be as in Example 1, with open unit ball $\Delta \times \Delta$. Fix $a \in \Delta \backslash\{0\}$. Define $h: \Delta \times \Delta \rightarrow \Delta \times \Delta$ by

$$
h(z, w)=\left(g_{a}(w), g_{-a}(z)\right) \quad(z, w \in \Delta)
$$

where $g_{a}(w)=\frac{a+w}{1+\bar{a} w}$. Then $\operatorname{Fix}(h)=\left\{\left(z, g_{-a}(z)\right): z \in \Delta\right\}$ which is not affine since $(0,-a)$ and $(a, 0)$ are in Fix $(h)$ while $\frac{1}{2}(0,-a)+\frac{1}{2}(a, 0) \notin \operatorname{Fix}(h)$. We also note that $h^{2 n}(z, w)=(z, w)$ and $h^{2 n+1}(z, w)=h(z, w)$. So $\left(h^{n}(z, w)\right)_{n}$ does not converge if $(z, w) \notin \operatorname{Fix}(h)$.

We are now ready to prove the Theorem. 
Proof of the Theorem. We will use the same symbol throughout for both a constant function and its value.

Let $\xi \in \partial B$ be the "Wolff point" of $f$ as mentioned in the beginning of this section. Let $\Gamma(f)$ be the set of all subsequential limits of $\left\{f^{n}: n=1,2, \ldots\right\}$ in $H(B, \bar{B})$ with respect to the topology of local uniform convergence. By Lemma 1 , $\Gamma(f) \neq \emptyset$.

We first show that $\Gamma(f)$ consists of constant maps only. Suppose, otherwise, that $\Gamma(f)$ contains a nonconstant map $g \in H(B, \bar{B})$. We deduce a contradiction. By Lemma 2, $g(B) \subset B$. Let $\left(f^{n_{k}}\right)$ be a subsequence of $\left(f^{n}\right)$ converging to $g$. Let $m_{k}=n_{k+1}-n_{k}$. By Remark $1,\left(f^{m_{k}}\right)$ has a convergent subsequence, and we may assume, without loss of generality, that $f^{m_{k}} \rightarrow h_{0} \in H(B, \bar{B})$. Since $f^{n_{k+1}}=f^{m_{k}} \circ f^{n_{k}} \rightarrow h_{0} \circ g$, we have $h_{0} \circ g=g$ and $h_{0}$ is the identity on $g(B)$. So $h_{0}$ is nonconstant, $h_{0}(B) \subset B$ and $A_{0}=\operatorname{Fix}\left(h_{0}\right)$ is an affine subset of $B$. Since $A_{0} \subset \overline{f(B)}$ which is compact, it follows that $\operatorname{dim} A_{0}<\infty$. Clearly, $A_{0} \subset h_{0}(B)$. If $A_{0} \neq h_{0}(B)$, then we repeat the above process. Letting $p_{k}=m_{k+1}-m_{k}$, we may assume $f^{p_{k}} \rightarrow h_{1} \in H(B)$ satisfying $h_{1} \circ h_{0}=h_{0}$. So $h_{1}$ is the identity on $h_{0}(B)$ and $h_{0}(B) \subset A_{1}=\operatorname{Fix}\left(h_{1}\right)$. We have $A_{0} \neq A_{1}$ and $A_{1}$ is a finite dimensional affine subset of $B$, with $\operatorname{dim} A_{1}>\operatorname{dim} A_{0}$. If $A_{1} \neq h_{1}(B)$, we repeat the process again. Continuing in this manner, we must eventually find some $h_{i} \in H(B)$ such that $h_{i}(B)=A_{i}=\operatorname{Fix}\left(h_{i}\right)$. For otherwise, we can construct a sequence $\left(v_{j}\right)_{j} \subset$ $\cup_{i=1}^{\infty} A_{i}$ with the property that $\left\|v_{i}-v_{j}\right\|>\delta$ for all $i \neq j$ and some $\delta>0$. Since $\cup_{i=1}^{\infty} A_{i} \subset \overline{f(B)}$ which is compact, this is clearly impossible. It follows that $h_{i}^{2}=h_{i}$. Let $\left(f^{l_{k}}\right)$ converge to $h_{i}$ locally uniformly. Note that $f\left(A_{i}\right) \subset A_{i}$. Since $A_{i}$ is a finite dimensional affine subset of $B$ and the automorphisms act transitively on $B$, a similar argument to that in [15, p. 98] shows that $A_{i}$ is biholomorphically equivalent to the open unit ball of $\mathbb{C}^{n}$ where $n=\operatorname{dim} A_{i}$. Now $\left.f\right|_{A_{i}}: A_{i} \rightarrow A_{i}$ is fixed-point-free and by $[13,15]$, there exists $\mu \in \partial A_{i}$ such that $\left(\left.f\right|_{A_{i}}\right)^{n}$ converges locally uniformly to $\mu$ on $A_{i}$. So $\left.h_{i}\right|_{A_{i}}=\lim _{k \rightarrow \infty}\left(\left.f\right|_{A_{i}}\right)^{l_{k}}=\mu$ which is impossible as $h_{i}$ is nonconstant and $h_{i}(B)=A_{i}$.

Therefore $\Gamma(f)$ must consist of constant maps only. Now take any $\eta \in \Gamma(f)$. Then $\eta \in \partial B$, for otherwise $\eta$ would be a fixed point of $f$ in $B$. There is a subsequence $\left(f^{n_{k}}\right)$ converging to $\eta$. Let $\lambda>0$ and let $z \in E(\xi, \lambda)$. We have

$$
\eta=\lim _{k \rightarrow \infty} f^{n_{k}}(z) \in \overline{E(\xi, \lambda)} \cap \partial B=\{\xi\}
$$

since $E(\xi, \lambda)$ is $f$-invariant. Therefore every convergent subsequence of $\left(f^{n}\right)$ converges to the constant map $\xi$. It follows from Remark 1 that $\left(f^{n}\right)$ must converge locally uniformly to $\xi$ and the proof is complete.

We end with the following example of a fixed-point-free compact holomorphic map on a Hilbert ball.

Example 3. Let $B$ be the open unit ball of the (complex) Hilbert space $l_{2}$. Define $f: B \longrightarrow B$ by

$$
\begin{aligned}
f\left(x_{1}, x_{2}, \ldots\right) & =\left(\frac{1+x_{1}}{2},\left(\frac{1-x_{1}}{2}\right) \frac{x_{1}}{2},\left(\frac{1-x_{1}}{2}\right) \frac{x_{2}}{3}, \ldots\right) \\
& =\left(\frac{1+x_{1}}{2}, 0,0, \ldots\right)+\frac{1-x_{1}}{2}\left(0, \frac{x_{1}}{2}, \frac{x_{2}}{3}, \ldots\right)
\end{aligned}
$$


for $\left(x_{1}, x_{2}, \ldots\right) \in B$. Then $f$ is fixed-point-free and holomorphic. Moreover, $f$ is compact since it is the sum of two compact maps. Also the Wolff point is $(1,0,0, \ldots)$.

\section{ACKNOWLEDGEMENTS}

This research was carried out during the authors' visits to each other's institution. We gratefully acknowledge financial support from the British Council/ Forbairt Joint Research Scheme and the Arts Faculty of University College Dublin.

\section{REFERENCES}

[1] M. Abate, Iteration theory, compactly divergent sequences and commuting holomorphic maps, Ann. della Scuola Normale Superiore di Pisa 18 (1991) 67-191. MR 93e:32034a

[2] R. B. Burckel, Iterating analytic self-maps of discs, Amer. Math. Monthly 88 (1981) 396-407. MR 82g:30046

[3] A. Denjoy, Sur l'itération des fonctions analytiques, C. R. Acad. Sc. Paris 182 (1926) 255-257.

[4] S. Dineen, "The Schwarz lemma", Oxford Univ. Press, Oxford, 1989. MR 91f:46064

[5] C. J. Earle and R. S. Hamilton, A fixed point theorem for holomorphic mappings, Proc. Symposia Pure Math 16 (1969) 61-65. MR 42:918

[6] M. P. Fatou, Sur l'équations fonctionnelles, Bull. Soc. Math. France 47 (1919) 161-271.

[7] M. P. Fatou, Sur l'équations fonctionnelles, Bull. Soc. Math. France 48 (1920) 22-94 and 208-314.

[8] K. Goebel, Fixed points and invariant domains of holomorphic mappings of the Hilbert ball, Nonlinear Analysis 6 (1982) 1327-1334. MR 84j:58027

[9] K. Goebel and S. Reich, "Uniform convexity, hyperbolic geometry and nonexpansive mappings", Marcel Dekker, 1984. MR 86d:58012

[10] K. Goebel and S. Reich, Iterating holomorphic self-mappings of the Hilbert ball, Proc. Japan. Acad. 58 (1982) 349-352. MR 84b:47065

[11] L. A. Harris, A generalization of $C^{*}$-algebras, Proc. London Math. Soc. 42 (1981) 331-361. MR 82e: 46089

[12] T. L. Hayden and T. J. Suffridge, Biholomorphic maps in Hilbert space have a fixed point, Pacific J. Math. 38 (1971) 419-422. MR 46:4288

[13] M. Hervé, Quelques propriétés des applications analytiques d'une boule à $m$ dimensions dans elle-meme, J. Math. Pures et Appl. 42 (1963) 117-147. MR 28:3177

[14] G. Julia, Mémoire sur l'itération des fonctions rationnelles, J. Math. Pures et Appl. 8 (1918) 47-245.

[15] B. D. MacCluer, Iterates of holomorphic self-maps of the open unit ball in $\mathbb{C}^{n}$, Michigan Math J. 30 (1983) 97-106. MR 85c:32047a

[16] P. Mellon, Another look at results of Wolff and Julia type for $J^{*}$-algebras, J. Math. Anal. Appl. 198 (1996) 444-457. CMP 96:08

[17] W. Rudin, "Function theory in the unit ball of $\mathbb{C}^{n}$ ", Springer, New York, 1980. MR 82i:32002

[18] A. Stachura, Iterates of holomorphic self-maps of the unit ball in Hilbert space, Proc. Amer. Math. Soc. 93 (1985) 88-90. MR 86b:47117

[19] H. Upmeier, "Symmetric Banach manifolds and Jordan $C^{*}$-algebras", North-Holland, 1985. MR 87a:58022

[20] E. Vesentini, Iteration of holomorphic maps, Russ. Math. Survey 40 (1985) 7-11.

[21] J. P. Vigué, Sur le groupe des automorphismes analytiques d'un ouvert borné d'un espace de Banach complexe, C. R. Acad. Sc. Paris 278 (1974) 617-620. MR 49:3230

[22] J. P. Vigué, Le groupe des automorphismes analytiques d'un domaine borné d'un espace de Banach complexe, application aux domaines bornés symétriques, Ann. Sc. Ec. Norm. Sup. 9 (1976) 203-282. MR 55:3340

[23] J. Wolff, Sur l'itération des fonctions bornées, C. R. Acad. Sc. Paris 182 (1926) 42-43 and 200-201. 
[24] J. Wolff, Sur une généralisation d'un théorème de Schwarz, C. R. Acad. Sc. Paris 182 (1926) 918-920 and 183 (1926) 500-502.

[25] K. Włodarczyk, Julia's lemma and Wolff's theorem for $J^{*}$-algebras, Proc. Amer. Math. Soc. 99 (1987) 472-476. MR 88a:46049

Goldsmiths College, University of London, London SE14 6NW, England

E-mail address: maa01chc@gold.ac.uk

Department of Mathematics, University College Dublin, Belfield, Dublin 4, Ireland

E-mail address: pmellon@irlearn.ucd.ie 